\title{
Learning Musculoskeletal Imaging
}

R. Ribes and J.C. Vilanova, eds.

New York, NY: Springer, 2010, 280 pages, $\$ 59.95$

This brief text on musculoskeletal imaging is a good, concise handbook of interesting cases compiled by many authors from Spain and showcasing a diverse spectrum of musculoskeletal diseases. The book is easy to read and will be helpful to medical students and radiology residents as a valuable introductory guide to the expanding and evolving field of musculoskeletal imaging. The 10 sections of the book cover infection and arthritis; tumors; tendons and muscles; bone marrow; spine; shoulder; elbow, hand, and wrist; hip and pelvis; knee; and ankle and foot as they pertain to musculoskeletal imaging. Each section has 10 illustrative case studies, with comments related to anatomic, physiopathologic, and radiologic viewpoints. This textbook provides a good introduction to musculoskeletal radiology, and the practical page layout with images on the left page and text and figure legends on the right page allows for convenient side-by-side visualization of the radiographic abnormality with the corresponding text. Most common and important topics are covered, affording one a good grasp of the basics of musculoskeletal radiology. MR images are provided in all cases, and plain radiographs in many cases, and these are also supplemented with multidetector CT scans, Doppler sonograms, or bone scans in selected cases and occasionally with gross or microscopic pathology and a photograph of the abnormality. The images are of excellent quality, and arrows point to the abnormality in most cases, making for easier identification. Each case begins with a brief relevant patient history followed by a half-page concise, relevant medical description of the disease process, comprising etiology, typical clinical and radiologic features, differential diagnosis, and the usefulness of the various imaging modalities. In addition, important histopathology and laboratory tests are briefly described in some of the case studies. The different therapeutic options are briefly mentioned, including the importance of telling the referring surgeons of special or important radiographic findings that may have an impact on the surgical management. Useful suggestions such as obtaining additional special views to showcase or better identify the radiographic abnormality are practically useful. Each case study concludes by clearly describing the relevant findings in the particular radiographic studies and providing a good list of references, Web links, and textbooks for additional reading. A narrow column at the right side of the page provides a convenient space to write notes. This book is not designed to show pathologic correlations, and it achieves its objective of informing us of the basics of musculoskeletal imaging.

Overall, this is a useful, informative, and easy-to-read and -understand book that uses the format of multiple case studies to cover imaging of a spectrum of musculoskeletal diseases. This book will serve as a good introduction to the field of musculoskeletal imaging. It conveniently combines the findings seen on high-quality musculoskeletal radiographic images with concise, adequate medical information on specific disease processes and will provide a strong and useful foundation to build on. This handy, practical reference guide will greatly benefit radiology residents and medical students as a quick, handy, go-to guide to learn and understand basic musculoskeletal imaging. Radiologists will find it useful for refreshing their knowledge about musculoskeletal imaging.

Usha A. Joseph

University of Texas Medical School at Houston

6431 Fannin, MSB $2.130 B$

Houston, TX 77030

E-mail: usha.a.joseph@uth.tmc.edu

COPYRIGHT (C 2011 by the Society of Nuclear Medicine, Inc. 